\title{
A New Class of Golden Riemannian Manifold
}

\author{
Gherici Beldjilali* \\ (Communicated by Kazım İlarslan)
}

\begin{abstract}
In this paper, we introduce a new class of almost Golden Riemannian structures and study their essential examples as well as their fundamental properties. Next, we investigate a particular type belonging to this class and we establish some basic results for Riemannian curvature tensor and the sectional curvature. Concrete examples are given.
\end{abstract}

\section{Introduction}

Manifolds equipped with certain differential-geometric structures possess rich geometric structures, such manifolds and relations between them have been studied widely in differential geometry. Indeed, almost complex manifolds, almost contact manifolds and almost product manifolds and relations between such manifolds have been studied extensively by many authors.

The differential geometry of the Golden on Riemannian manifolds is a popular subject for mathematicians. In 2007, Hretcanu [9] introduced the Golden structure on manifolds. In a recent paper of Crasmareanu and Hretcanu [5], the geometry of the Golden structure on manifolds have studied. In particular, they have investigated the geometry of the Golden structure on a manifold by using the corresponding almost product structure.

In [7], Gezer, Cengiz and Salimov have studied the Riemannian manifolds endowed with Golden structures and they have given important theorems for these manifolds. Recently, many several works are dealing with this subject [1], [2], [6], [10], [11].

The aim of this work, is to prove the existence of Golden Riemannian structures that are integrable with structural tensor $\Phi$ witch is not covariantly constant with respect to the Levi-Civita connection and we study their fundamental properties.

The paper is organized as follows:

In $\S 2$ we recall some definitions and properties of the Golden structure.

The $\S 3$ is devoted to the study of such a class of almost Golden Riemannian manifolds where we give a new sufficient condition for the integrability, we prove the existence of almost Golden Riemannian structures that are integrable with $\nabla \Phi \neq 0$ where $\nabla$ is the Levi-Civita connection and we construct concrete example.

The $\S 4$ is intended to study a more special type in this class by giving their geometric properties and we deal with curvature tensors of this special type. 


\section{Almost Golden Riemannian structures}

Let $(M, g)$ be a Riemannian manifold. A Golden structure on $(M, g)$ is a non-null tensor field $\Phi$ of type $(1,1)$ which satisfies the equation

$$
\Phi^{2}=\Phi+I
$$

where $I$ is the identity transformation.

Remark 2.1. A Golden structure $\Phi$ is an isomorphism on the tangent space of the manifold, $T_{p} M$, for every $p \in M$ and the eigenvalues of $\Phi$ are the Golden ratio $\phi=\frac{1+\sqrt{5}}{2}$ and $\phi^{*}=\frac{1-\sqrt{5}}{2}=1-\phi$.

We say that the metric $g$ is $\Phi$ compatible if

$$
g(\Phi X, Y)=g(X, \Phi Y)
$$

for all $X, Y$ vectors fields on $M$.

If we substitute $\Phi X$ into $X$ in (2.2), equation (2.2) may also be written as

$$
g(\Phi X, \Phi Y)=g\left(\Phi^{2} X, Y\right)=g((\Phi+I) X, Y)=g(\Phi X, Y)+g(X, Y) .
$$

The Riemannian metric (2.2) is called $\Phi$-compatible and $(M, \Phi, g)$ is called an almost Golden Riemannian manifold [5].

Unlike the papers [5], [9], [7] and in support of what was stated in [6] we use the word "almost" to general Golden structures on a manifold. We say that a manifold $M$ is a Golden manifold if it has an integrable almost Golden structure. Recall that the structure $\Phi$ is integrable if the Nijenhuis tensor $N_{\Phi}$ vanishes, where

$$
N_{\Phi}(X, Y)=\Phi^{2}[X, Y]+[\Phi X, \Phi Y]-\Phi[\Phi X, Y]-\Phi[X, \Phi Y]
$$

\section{Almost Golden Riemannian almost contact metric structure}

Let $(M, \Phi, g)$ be a $n$-dimensional Golden Riemannian manifold. For every $p \in M$ the tangent vector space $T_{p} M$ splits as follows $T_{p} M=\left(D_{\phi^{*}}\right)_{p} \oplus\left(D_{\phi}\right)_{p}$, where $\phi=\frac{1+\sqrt{5}}{2}, \phi^{*}=\frac{1-\sqrt{5}}{2}=1-\phi$ and

$$
\left(D_{\phi^{*}}\right)_{p}=\left\{\xi \in T_{p} M: \Phi_{p} \xi=\phi^{*} \xi\right\}, \quad\left(D_{\phi}\right)_{p}=\left\{\psi \in T_{p} M: \Phi_{p} \psi=\phi^{*} \psi\right\} .
$$

Then one has the next result.

Proposition 3.1. [6] Let $(M, \Phi, g)$ be a n-dimensional Golden Riemannian manifold. The G-structure over $M$ defined by $(\Phi, g)$ is

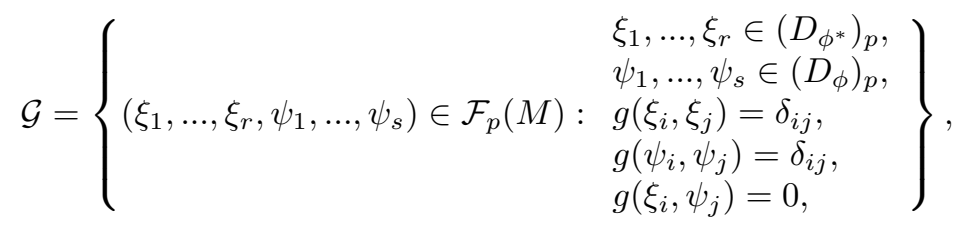

whose structural group is $G=O(r ; \mathbf{R}) \times O(s ; \mathbf{R}), \mathcal{F}(M)$ is the principal bundle of linear frames of $M, r=\operatorname{dim}_{\phi^{*}}, s=$ $\operatorname{dim} D_{\phi}$ and $r+s=n$.

Let $U \subseteq M$ be an open subset. An adapted local frame to the $G$-structure defined by $(\Phi, g)$ in $U$ is a family $\left(\xi_{1}, \ldots, \xi_{r}, \psi_{1}, \ldots, \psi_{s}\right) \in U$ where $\xi_{1}, \ldots, \xi_{r}, \psi_{1}, \ldots, \psi_{s} \in \mathcal{X}(U)$ such that $\left(\xi_{1}(p), \ldots, \xi_{r}(p), \psi_{1}(p), \ldots, \psi_{s}(p)\right) \in \mathcal{G}, \forall p \in U$. Its dual local frame is the family $\left(\eta_{1}, \ldots, \eta_{r}, \omega_{1}, \ldots, \omega_{s}\right) \in U$ where $\eta_{1}, \ldots, \eta_{r}, \omega_{1}, \ldots, \omega_{s} \in \bigwedge^{1}(U)$, satisfying

$$
\eta_{i}(X)=g\left(\xi_{i}, X\right), \quad \psi_{j}(X)=g\left(\psi_{j}, X\right), i=1, \ldots, r, j=1, \ldots, s, \forall X \in \mathcal{X}(U)
$$

Thus, the almost Golden structure $\Phi$ always can be written locally in $U$ as follows:

$$
\left.\Phi\right|_{U}=\phi I-\sqrt{5} \sum_{i=1}^{r} \eta_{i} \otimes \xi_{i}=\phi^{*} I+\sqrt{5} \sum_{j=1}^{s} \omega_{j} \otimes \psi_{j},
$$

www.iejgeo.com 
Notice that

$$
I=\sum_{i=1}^{r} \eta_{i} \otimes \xi_{i}+\sum_{j=1}^{s} \omega_{j} \otimes \psi_{j} .
$$

If the almost Riemannian manifold $(M, \Phi, g)$ satisfies $r=1$ then the almost Golden structure $\Phi$ can be written locally in $U$ according to the above notation as follows:

$$
\left.\Phi\right|_{U}=\phi I-\sqrt{5} \eta \otimes \xi,
$$

where $\xi=\xi_{1}$ and $\eta=\eta_{1}$.

The first subject of this paper is to study a new class of almost Golden Riemannian manifolds that is integrable despite $\nabla \Phi \neq 0$ where $\nabla$ is the Levi-Civita connection.

Definition 3.1. Let $M$ be a differentiable manifold of dimension $2 n+1$. An almost Golden Riemannian almost contact metric structure on $M$ is the data $(\Phi, \xi, \eta, g)$ where:

$\xi$ is a global vectors field.

$\eta$ is a differential 1-form on $M$ such that $\eta(\xi)=1$.

$g$ is a Riemannian metric such that $g(X, \xi)=\eta(X)$.

$\Phi$ is a tensor field of type $(1,1)$ satisfying

$$
\Phi=\phi I-\sqrt{5} \eta \otimes \xi,
$$

for all vectors field $X$ on $M$.

In addition, if $\Phi$ is integrable then, $(\Phi, \xi, \eta, g)$ is an Golden Riemannian almost contact metric structure and $(M, \Phi, \xi, \eta, g)$ is called a Golden Riemannian almost contact metric manifold.

Proposition 3.2. Let $(M, \Phi, \xi, \eta, g)$ be an almost Golden Riemannian almost contact metric manifold and $U$ be a coordinate neighborhood on $M$ and $\psi_{i}$ any unitary vectors fields on $U$ such that $\Phi \psi_{i}=\phi \psi_{i}$ where $i \in\{1, \ldots, 2 n\}$. Then, we may easily check that the set $\left\{\xi, \psi_{i}\right\}$ is a local orthonormal basis on $M$.

We refer to this basis as Golden basis.

We define The fundamental 2-form $\Omega$ by:

$$
\begin{aligned}
\Omega(X, Y) & =g\left(X, \sum_{j=1}^{n}\left(\psi_{2 j-1} \wedge \psi_{2 j}\right) Y\right) \\
& =g\left(X, \sum_{j=1}^{n} \omega_{2 j}(Y) \psi_{2 j-1}-\omega_{2 j-1}(Y) \psi_{2 j}\right) \\
& =\sum_{j=1}^{n}\left(\omega_{2 j}(Y) \omega_{2 j-1}(X)-\omega_{2 j-1}(Y) \omega_{2 j}(X)\right) \\
& =2 \sum_{j=1}^{n}\left(\omega_{2 j-1} \wedge \omega_{2 j}\right)(X, Y),
\end{aligned}
$$

for all vectors fiels $X, Y$ on $M$ with $(X \wedge Y) Z=g(Y, Z) X-g(X, Z) Y$.

For brevity, we put

$$
\varphi=\sum_{j=1}^{n}\left(\psi_{2 j-1} \wedge \psi_{2 j}\right),
$$

we obtain

$$
\Omega(X, Y)=g(X, \varphi Y),
$$

using the formula (3.2), we note that

$$
\begin{aligned}
\varphi^{2} X & =-\sum_{j=1}^{n}\left(\omega_{2 j-1}(X) \psi_{2 j-1}+\omega_{2 j}(X) \psi_{2 j}\right) \\
& =-X+\eta(X) \xi
\end{aligned}
$$


That is why, this class of structures is called almost Golden Riemannian almost contact structure. For more details on almost contact structures see ([3], [4], [12]).

Proposition 3.3. Let $(M, \Phi, \xi, \eta, g)$ be an almost Golden Riemannian almost contact structure. Then, we have

$$
\mathrm{d} \Omega=\frac{\alpha}{n} \eta \wedge \Omega
$$

where d denote the exterior derivative and

$$
\alpha=\operatorname{div} \xi+\sum_{i<j}\left(\left(\mathcal{L}_{\xi} g\right)\left(\psi_{2 i-1}, \psi_{2 j-1}\right)+\left(\mathcal{L}_{\xi} g\right)\left(\psi_{2 i}, \psi_{2 j}\right)\right)
$$

Proof. Let $U=\sum_{i=1}^{n} \psi_{2 i-1}$ and $V=\sum_{i=1}^{n} \psi_{2 i}$ two vectors fields on $M$. Putting $\mathrm{d} \Omega=\sigma \eta \wedge \Omega$ for a certain functions $\sigma$ on $M$. Then, we get

$$
\begin{aligned}
& 3(\eta \wedge \Omega)(\xi, U, V)=n, \\
3 \mathrm{~d} \Omega(\xi, U, V)= & \left(\nabla_{\xi} \Omega\right)(U, V)+\left(\nabla_{U} \Omega\right)(V, \xi)+\left(\nabla_{V} \Omega\right)(\xi, U) \\
= & -\Omega\left(V, \nabla_{U} \xi\right)-\Omega\left(\nabla_{V} \xi, U\right) \\
= & \sum_{j=1}^{n}\left(\omega_{2 j-1}\left(\nabla_{U} \xi\right)+\omega_{2 j}\left(\nabla_{V} \xi\right)\right) \\
= & g\left(\nabla_{U} \xi, U\right)+g\left(\nabla_{V} \xi, V\right) \\
= & \operatorname{div} \xi+\sum_{i<j}\left(\left(\mathcal{L}_{\xi} g\right)\left(\psi_{2 i-1}, \psi_{2 j-1}\right)+\left(\mathcal{L}_{\xi} g\right)\left(\psi_{2 i}, \psi_{2 j}\right)\right) \\
= & \alpha,
\end{aligned}
$$

which implies $\sigma=\frac{\alpha}{n}$.

Corollary 3.1. Let $M$ be 3-dimensional almost Golden Riemannian almost contact structure. Then, we have

$$
\mathrm{d} \Omega=\operatorname{div} \xi \eta \wedge \Omega .
$$

Now, we shall introduce a new sufficient condition for the integrability of this class of structures.

Theorem 3.1. Let $(M, \Phi, \xi, \eta, g)$ be an almost Golden Riemannian almost contact structure. If $\eta$ is closed then $\Phi$ is integrable.

Proof. Using the formulas (2.3) and (3.2) we can get

$$
\begin{aligned}
N_{\Phi}(X, Y) & =-10(\mathrm{~d} \eta(X, Y)+\eta(X) \mathrm{d} \eta(\xi, Y)+\eta(Y) \mathrm{d} \eta(X, \xi)) \xi \\
& =-10 \mathrm{~d} \eta\left(\varphi^{2} X, \varphi^{2} Y\right) \xi .
\end{aligned}
$$

So, easily we can see that for $\mathrm{d} \eta=0$ we obtain $N_{\Phi}=0$.

The main purpose of [7] was to characterize the integrability of almost Golden structures on almost Golden Riemannian manifolds. Proposition 3.4 is the most known characterization of the integrability of the polynomial structure $\Phi$ on an almost Golden Riemannian manifold $(M, \Phi, g)$ (see, e.g. [7]). In the quoted paper, the authors have obtained another sufficient condition to the integrability of $\Phi$, which is not a necessary condition, they have showed that if $\nabla \Phi=0$ with $\nabla$ is the Levi-Civita connection of $g$ then, the almost Golden Riemannian structure $\Phi$ is integrable. It led to ask if there exist a Golden Riemannian structure with $\nabla \Phi \neq 0$ ? The answer is positive according to the following example:

Example 3.1. We denote the Cartesian coordinates in a 3-dimensional Euclidean space $E^{3}$ by $(x, y, z)$ and define a symmetric tensor field $g$ by

$$
g=\left(\begin{array}{ccc}
\rho(x, y, z)^{2}+\tau(x, y, z)^{2} & 0 & -\tau(x, y, z) \\
0 & \rho(x, y, z)^{2} & 0 \\
-\tau(x, y, z) & 0 & 1
\end{array}\right)
$$

www.iejgeo.com 
where $\rho$ and $\tau$ are two functions on $E^{3}$.

Further, we define an almost Golden structure $\Phi$ on $E^{3}$ by:

$$
\Phi=\left(\begin{array}{ccc}
\phi & 0 & 0 \\
0 & \phi & 0 \\
\tau(x, y, z) \sqrt{5} & 0 & \phi^{*}
\end{array}\right) .
$$

One can easily proof that for all vector fields $X$ and $Y$ on $E^{3}$ we have

$$
\Phi^{2} X=\Phi X+X \quad \text { and } \quad g(\Phi X, Y)=g(X, \Phi Y),
$$

then, $(\Phi, g)$ is an almost Golden Riemannian structure.

The almost Golden structure $\Phi$ admit $\xi=\frac{\partial}{\partial z}$ as the unitary eigenvector associated with $\phi^{*}$ and $\psi_{1}=$ $\frac{1}{\rho}\left(\frac{\partial}{\partial x}+\tau \frac{\partial}{\partial z}\right), \psi_{2}=\frac{1}{\rho} \frac{\partial}{\partial y}$ the other two unit eigenvectors associated with $\phi$. The 1-forms corresponding to $\xi, \psi_{1}$ and $\psi_{2}$ respectively are $\eta=-\tau d x+d z, \omega_{1}=\rho d x$ and $\omega_{2}=\rho d y$.

We notice that

$$
\Phi=\phi I-\sqrt{5} \eta \otimes \xi=\phi^{*} I+\sqrt{5}\left(\omega_{1} \otimes \psi_{1}+\omega_{2} \otimes \psi_{2}\right),
$$

and $\left\{\xi, \psi_{1}, \psi_{2}\right\}$ is the Golden basis.

In addition,

$$
\mathrm{d} \eta=\tau_{2} d x \wedge d y+\tau_{3} d x \wedge d z, \quad \text { with } \quad \tau_{i}=\frac{\partial \tau}{\partial x_{i}},
$$

so, if $\tau_{2}=\tau_{3}=0$ then, $\Phi$ is integrable in which case $\left(E^{3}, \Phi, \xi, \eta, g\right)$ is a Golden Riemannian almost contact metric manifold.

We can find

$$
\left[\xi, \psi_{1}\right]=-\frac{\rho_{3}}{\rho} \psi_{1}, \quad\left[\xi, \psi_{2}\right]=-\frac{\rho_{3}}{\rho} \psi_{2}, \quad\left[\psi_{1}, \psi_{2}\right]=\frac{\rho_{2}}{\rho^{2}} \psi_{1}-\frac{\rho_{1}+\tau \rho_{3}}{\rho^{2}} \psi_{2}
$$

Using Koszul's formula for the metric $g$

$$
\begin{aligned}
2 g\left(\nabla_{X} Y, Z\right) & =X g(Y, Z)+Y g(Z, X)-Z g(X, Y) \\
& -g(X,[Y, Z])+g(Y,[Z, X])+g(Z,[X, Y],
\end{aligned}
$$

we obtain:

$$
\begin{gathered}
\nabla_{\xi} \xi=\nabla_{\xi} \psi_{1}=\nabla_{\xi} \psi_{2}=0, \\
\nabla_{\psi_{1}} \xi=\frac{\rho_{3}}{\rho} \psi_{1}, \quad \nabla_{\psi_{1}} \psi_{1}=-\frac{\rho_{3}}{\rho} \xi-\frac{\rho_{2}}{\rho} \psi_{2}, \quad \nabla_{\psi_{1}} \psi_{2}=\frac{\rho_{2}}{\rho} \psi_{1}, \\
\nabla_{\psi_{2}} \xi=\frac{\rho_{3}}{\rho} \psi_{2}, \quad \nabla_{\psi_{2}} \psi_{1}=\frac{\rho_{1}+\tau \rho_{3}}{\rho^{2}} \psi_{2}, \quad \nabla_{\psi_{2}} \psi_{2}=-\frac{\rho_{3}}{\rho} \xi-\frac{\rho_{1}+\tau \rho_{3}}{\rho^{2}} \psi_{1} .
\end{gathered}
$$

finally, knowing that

$$
\left(\nabla_{X} \Phi\right) Y=\nabla_{X} \Phi Y-\Phi \nabla_{X} Y
$$

the non zero components of $\nabla \Phi$ are:

$$
\begin{gathered}
\left(\nabla_{\psi_{1}} \Phi\right) \xi=-\sqrt{5} \frac{\rho_{3}}{\rho} \psi_{1}, \quad\left(\nabla_{\psi_{2}} \Phi\right) \xi=-\sqrt{5} \frac{\rho_{3}}{\rho} \psi_{2}, \\
\left(\nabla_{\psi_{1}} \Phi\right) \psi_{1}=\left(\nabla_{\psi_{2}} \Phi\right) \psi_{2}=-\sqrt{5} \frac{\rho_{3}}{\rho} \xi
\end{gathered}
$$

where $\rho_{i}=\frac{\partial \rho}{\partial x_{i}}$. For $\rho_{3} \neq 0$ we notice that $\nabla \Phi \neq 0$.

In the rest of this paper, we examine a special type in this class by giving their geometric properties and we deal with the curvature tensors.

Lemma 3.1. Let $(M, \Phi, \xi, \eta, g)$ be a Golden Riemannian almost contact manifold. Then,

$$
\nabla_{\xi} \xi=0
$$

(equivalently the integral curves of $\xi$ are geodesics). 
Proof. Since $\eta$ is closed then,

$$
\begin{aligned}
2 \mathrm{~d} \eta(X, Y) & =0 \\
& =g\left(\nabla_{X} \xi, Y\right)-g\left(\nabla_{Y} \xi, X\right),
\end{aligned}
$$

putting $X=\xi$ we obtain $\nabla_{\xi} \xi=0$.

In view of the new condition of integrability we state an interesting type of almost Golden Riemannian almost contact metric manifolds. Briefly, we denote such a type by Golden* manifold.

\section{Golden* manifold}

Definition 4.1. A Golden* manifold is an almost Golden Riemannian almost contact manifold $(M, \Phi, \xi, \eta, g)$ which satisfies:

$$
\left(\nabla_{X} \Phi\right) Y=\sqrt{5}(g(X, Y) \xi+\eta(Y) X-2 \eta(X) \eta(Y) \xi),
$$

for all $X, Y$ vectors fields on $M$.

Remark 4.1. This type has been introduced in [2] under the name "G-Golden manifold" for a manifold of any dimension, but here the dimension is odd.

Lemma 4.1. Any Golden* manifold is a Golden Riemannian manifold.

Proof. it is sufficient to prove that $\mathrm{d} \eta=0$. Using the formula (4.1), we obtain

$$
\left(\nabla_{X} \Phi\right) \xi=\sqrt{5}(X-\eta(X) \xi)
$$

on the other hand, the formula (3.2) give

$$
\left(\nabla_{X} \Phi\right) \xi=-\sqrt{5} \nabla_{X} \xi
$$

from (4.2) and (4.3) we obtain

$$
\nabla_{X} \xi=-X+\eta(X) \xi
$$

and since $2 \mathrm{~d} \eta(X, Y)=g\left(\nabla_{X} \xi, Y\right)-g\left(\nabla_{Y} \xi, X\right)$ then $\mathrm{d} \eta=0$.

Example 4.1. Using the data of the above example (3.1), we have $\left(E^{3}, \Phi, \xi, \eta, g\right)$ is a Golden Riemannian almost contact manifold with $\tau_{2}=\tau_{3}=0$.

The non zero components of the tensor

$$
T(X, Y)=\sqrt{5}(g(X, Y) \xi+\eta(Y) X-2 \eta(X) \eta(Y) \xi)
$$

are:

$$
T\left(\psi_{1}, \xi\right)=\sqrt{5} \psi_{1}, \quad T\left(\psi_{2}, \xi\right)=\sqrt{5} \psi_{2}, \quad T\left(\psi_{1}, \psi_{1}\right)=T\left(\psi_{2}, \psi_{2}\right)=\sqrt{5} \xi,
$$

considering the components of $\nabla \Phi$ (see example 3.1), we conclude that $\left(E^{3}, \Phi, \xi, \eta, g\right)$ is a Golden* manifold if $\rho_{3}=-\rho$.

Let $(M, \Phi, \xi, \eta, g)$ be a Golden* manifold. First of all, we prepare:

\section{Proposition 4.1.}

$$
\begin{gathered}
R(X, Y) \xi=\eta(X) Y-\eta(Y) X, \\
R(X, \xi) Y=g(X, Y) \xi-\eta(Y) X, \\
S(X, \xi)=(1-n) \eta(X),
\end{gathered}
$$

where $S$ denote the Ricci curvature and $R$ is the curvature tensor defined by

$$
R(X, Y) Z=\nabla_{X} \nabla_{Y} Z-\nabla_{Y} \nabla_{X} Z-\nabla_{[X, Y]} Z .
$$


Proof. The relation (4.5) follows from (4.8) with $Z=\xi$. For the second relation, we have for all vectors fields $X, Y, Z$ on $M$

$$
g(R(X, \xi) Y, Z)=-g(R(Y, Z) \xi, X)
$$

and using (4.5). finally, knowing that

$$
S(X, Y)=\sum_{i=1}^{n} g\left(R\left(X, e_{i}\right) e_{i}, Y\right)
$$

where $\left\{e_{i}\right\}$ is a local orthonormal frame field, then

$$
S(X, \xi)=-\sum_{i=1}^{n} g\left(R\left(X, e_{i}\right) \xi, e_{i}\right)
$$

using (4.5) we obtain (4.7). This completes the proof of the proposition.

Let $X$ be the unit vector field orthogonal to $\xi$. Then, by use of (4.6), we obtain

$$
R(X, \xi, X, \xi)=1 \text {. }
$$

Thus we have:

Proposition 4.2. On a Golden* manifold, the sectional curvature of all plane section containing $\xi$ is 1.

Proposition 4.3.

$$
\begin{gathered}
\Phi R(X, Y) Z=\phi R(X, Y) Z+\sqrt{5}(\eta(X) g(Y, Z) \xi-\eta(Y) g(X, Z) \xi), \\
R(\Phi X, Y) Z=\phi R(X, Y) Z+\sqrt{5}(\eta(X) g(Y, Z) \xi-\eta(X) \eta(Z) Y), \\
R(X, \Phi Y) Z=\phi R(X, Y) Z+\sqrt{5}(\eta(Y) \eta(Z) X-\eta(Y) g(X, Z) \xi), \\
R(X, Y) \Phi Z=\phi R(X, Y) Z+\sqrt{5}(\eta(Y) \eta(Z) X-\eta(X) \eta(Z) Y), \\
S(\Phi X, Y)=S(X, \Phi Y) .
\end{gathered}
$$

Proof. Obvious, we just use the formula (3.2) and the proposition 4.1.

Proposition 4.4. If the sectional curvature of any Golden* manifold is a constant $c$ then, $c=-1$.

Proof. Let $(M, \Phi, \xi, \eta, g)$ be a Golden* manifold of constant sectional curvature $c$, i.e. we have

$$
R(X, Y) Z=c(X \wedge Y) Z
$$

Using the formula (4.10), we obtain

$$
R\left(\Phi X, e_{i}\right) e_{i}=\phi R\left(X, e_{i}\right) e_{i}+2 n \sqrt{5} \eta(X) \xi
$$

and since $R\left(X, e_{i}\right) e_{i}=2 n c X$, then from (4.15) we get $c=-1$.

Now, let $\mathcal{P}$ be a plane section in tangent space $T_{p} M$ at a point $p$ of $M$ and let it be spanned by vectors $X$ and $Y$. Then, the sectional curvature of $\mathcal{P}$ is given by

$$
K(X, Y)=\frac{R(X, Y, X, Y)}{g(X, X) g(Y, Y)-g(X, Y)^{2}} .
$$

On an almost Golden Riemannian manifold $M$, the section $\mathcal{P}=\{X, \Phi X\}$ where $X$ is a unit vector fields and $g(X, \Phi X)=\frac{1}{2}$ is called Golden section and the curvature associated with this section is called Golden sectional curvature which is denoted by $H(X)$ where

$$
H(X)=\frac{4}{5} R(X, \Phi X, X, \Phi X)
$$


Proposition 4.5. If $(M, \Phi, \xi, \eta, g)$ is an almost Golden Riemannian almost contact manifold then, the Golden sectional curvature $H_{\xi}(X)$ is given by

$$
H_{\xi}(X)=2 R(X, \xi, X, \xi) \text {. }
$$

Proof. Using the formula (4.16) with $\Phi X=\phi X-\sqrt{5} \eta(X) \xi$, we find

$$
H(X)=5 \eta(X)^{2} R(X, \xi, X, \xi),
$$

and the condition $g(X, \Phi X)=\frac{1}{2}$ gives $\eta(X)=\frac{1}{\sqrt{2}}$ and this completes the proof.

Proposition 4.6. On a Golden* manifold, the Golden sectional curvature is 2.

Proof. The proof of this result is a direct consequence of propositions (4.1) and (4.5).

\section{References}

[1] Beldjilali, G.: Induced Structures on Golden Riemannian Manifolds. Beitr Algebra Geom. 59 (4), $761-777$ (2018).

[2] Beldjilali, G.: s-Golden manifolds, Mediterr. J. Math. (2019). https://doi.org/10.1007/s00009-019-1343-9.

[3] Blair, D. E.: Riemannian Geometry of Contact and Symplectic Manifolds. Progress in Mathematics, Vol. 203, Birhauser, Boston, (2002).

[4] Boyer, C.P., Galicki, K., Matzeu, P.: On Eta-Einstein Sasakian Geometry. Comm.Math. Phys. 262, 177-208 (2006).

[5] Crasmareanu, M., Hretecanu C.E.: Golden differential geometry. Chaos, Solitons \& Fractals. 38, 1124-1146 (2008).

[6] Etayo, F., Santamaria R., Upadhyay, A.: On the Geometry of Almost Golden Riemannian Manifolds, Mediterr. J. Math. 14,14-187 (2017). doi 10.1007/s00009-017-0991-x.

[7] Gezer, A., Cengiz N., Salimov, A.: On integrability of Golden Riemannian structures. Turkish J.Math. 37, 693-703 (2013).

[8] Gezer, A., Karaman, C.: Golden-Hessian Structures. Proc. Nat. Acad. Sci. 86, 41-46 (2016).

[9] Hretcanu, C. E.: Submanifolds in Riemannian manifold with Golden structure. In: Workshop on Finsler Geometry and its Applications, Hungary (2007).

[10] Ozkan, M., Yilmaz, F.: Prolongation of Golden structures to tangent bundles of order r. Commun. Fac. Sci. Univ. Ank. Sér. A1 Math. Stat. Volume 65 (1), 35-47 (2016).

[11] Sahin, B., Akyol, M. A.: Golden maps between Golden Riemannian manifolds and constancy of certain maps. Math. Commun. 19, 333-342 (2014).

[12] Yano, K., Kon, M.: Structures on Manifolds. Series in Pure Math., Vol 3, World Sci., (1984).

\section{Affiliations}

\section{GHERICI BELDJILALI}

ADDRESS: Laboratory of Quantum Physics and Mathematical Modeling (LPQ3M), University of Mascara, Algeria.

E-MAIL: gherici.beldjilali@univ-mascara.dz

\section{ORCID ID: 0000-0002-8933-1548}

